\title{
Brain Structure and Function Associated with Younger Adults in Growth Hormone Receptor-Deficient Humans
}

\author{
Kaoru Nashiro, ${ }^{1 *}$ Jaime Guevara-Aguirre, ${ }^{2,3 *}$ Meredith N. Braskie, ${ }^{4 *}$ (CGeorge W. Hafzalla, ${ }^{4}$ Rico Velasco, ${ }^{1}$ \\ Priya Balasubramanian, ${ }^{1}$ Min Wei, ${ }^{1}$ Paul M. Thompson, ${ }^{4}$ Mara Mather, ${ }^{1}$ Marvin D. Nelson, ${ }^{5}$ Alexandra Guevara, ${ }^{3}$ \\ - $D$ Enrique Teran, ${ }^{2}$ and Valter D. Longo ${ }^{1,6,7,8}$ \\ ${ }^{1}$ Leonard Davis School of Gerontology, University of Southern California, Los Angeles, California 90089, 2niversidad San Francisco de Quito, Quito, \\ Ecuador 170157, ${ }^{3}$ Institute of Endocrinology, Metabolism, and Reproduction, Quito, Ecuador 170523, ${ }^{4}$ Imaging Genetics Center, Keck School of Medicine, \\ University of Southern California, Marina del Rey, California 90292, 5 Department of Radiology, Children's Hospital Los Angeles/Keck School of Medicine of \\ University of Southern California, Los Angeles, California 90027, ${ }^{6}$ Longevity Institute, University of Southern California, Los Angeles, California 90089, \\ ${ }^{7}$ Department of Biological Sciences, University of Southern California, Los Angeles, California 90089, and ${ }^{8}$ FIRC (Italian Foundation for Cancer Research) \\ Institute of Molecular Oncology, Milano, Italy 20139
}

Growth hormone receptor deficiency (GHRD) results in short stature, enhanced insulin sensitivity, and low circulating levels of insulin and insulin-like growth factor 1 (IGF-1). Previous studies in mice and humans suggested that GHRD has protective effects against age-related diseases, including cancer and diabetes. Whereas GHRD mice show improved age-dependent cognitive performance, the effect of GHRD on human cognition remains unknown. Using MRI, we compared brain structure, function, and connectivity between 13 people with GHRD and 12 unaffected relatives. We assessed differences in white matter microstructural integrity, hippocampal volume, subregional volumes, and cortical thickness and surface area of selected regions. We also evaluated brain activity at rest and during a hippocampal-dependent pattern separation task. The GHRD group had larger surface areas in several frontal and cingulate regions and showed trends toward larger dentate gyrus and $\mathrm{CA} 1$ regions of the hippocampus. They had lower mean diffusivity in the genu of the corpus callosum and the anterior thalamic tracts. The GHRD group showed enhanced cognitive performance and greater task-related activation in frontal, parietal, and hippocampal regions compared with controls. Furthermore, they had greater functional synchronicity of activity between the precuneus and the rest of the default mode network at rest. The results suggest that, compared with controls, GHRD subjects have brain structure and function that are more consistent with those observed in younger adults reported in previous studies. Further investigation may lead to improved understanding of underlying mechanisms and could contribute to the identification of treatments for age-related cognitive deficits.

Key words: aging; cognition; growth hormone receptor deficiency; IGF-1; insulin; MRI

\section{Significance Statement}

People and mice with growth hormone receptor deficiency (GHRD or Laron syndrome) are protected against age-related diseases including cancer and diabetes. However, in humans, it is unknown whether cognitive function and brain structure are affected by GHRD. Using MRI, we examined cognition in an Ecuadorian population with GHRD and their unaffected relatives. The GHRD group showed better memory performance than their relatives. The differences in brain structure and function that we saw between the two groups were not consistent with variations typically associated with brain deficits. This study contributes to our understanding of the connection between growth genes and brain aging in humans and provides data indicating that GHR inhibition has the potential to protect against age-dependent cognitive decline.

\section{Introduction}

Growth hormone receptor deficiency (GHRD) in humans is caused by mutations in the GHR gene that lead to both insulin

Received June 14, 2016; revised Dec. 15, 2016; accepted Dec. 19, 2016.

Author contributions: K.N., J.G.-A., M.N.B., P.M.T., M.M., and V.D.L. designed research; K.N., J.G.-A., M.N.B., R.V., P.B., M.W., A.G., and E.T. performed research; K.N., M.N.B., G.W.H., M.W., and M.D.N. analyzed data; K.N., J.G.-A., M.N.B., G.W.H., P.B., M.W., P.M.T., M.M., M.D.N., and V.D.L. wrote the paper.

This work was supported by the National Institutes of Health (Grant AG P01AG034906 to V.D.L.; Grant R01 AG041915 to P.M.T.; Grant P50 AG05142 to Principal Investigator Dr. Helena Chui (Department of Neurology, USC
Keck School of Medicine) and M.N.B.; and Grant R01 AG025340 to M.M.). V.D.L. has equity interest in DSR Pharmaceuticals, a company developing longevity drugs. The remaining authors declare no competing financial interests. K.N., J.G.-A., and M.N.B. contributed equally to this work. We thank Allison Ponzio, Ph.D., and Tae-Ho Lee, Ph.D., for originally programming the pattern separation task.

Correspondence should be addressed to either of the following: Valter D. Longo, Ph.D., Leonard Davis School of Gerontology, University of Southern California, 3715 McClintock Ave., Los Angeles, CA 90089, E-mail: vlongo@usc.edu; or Jaime Guevara-Aguirre, Universidad San Francisco de Quito USFQ, Av. Diego de Robles y Pampite, Cumbaya Quito, Ecuador 170157, E-mail: jguevara@usfq.edu.ec.

DOI:10.1523/JNEUROSCI.1929-16.2016

Copyright $\odot 2017$ the authors $\quad 0270-6474 / 17 / 371696-12 \$ 15.00 / 0$ 
and insulin-like growth factor 1 (IGF-1) deficiency. GHR signaling is responsible for $\sim 90 \%$ of circulating levels of IGF-1 (Zhou et al., 1997), a factor that affects brain structure and function during development and aging. IGF-1 stimulates adult hippocampal neurogenesis in rodents and increased IGF-1 levels have been associated with improved spatial learning in mice (Trejo et al., 2008). Lower blood IGF-1 levels are associated with worse cognition in healthy older adults (Deijen et al., 2011) and IGF-1 also protects against amyloid accumulation and amyloidinduced cell death such as that found in Alzheimer's disease (AD) (Carro et al., 2002). However, recent reports have proposed that, as for insulin, chronically high levels of IGF-1 can induce IGF-1 resistance, which may contribute to neurodegeration (Spielman et al., 2014). Indeed, our recent work has shown that periodic cycles of restriction in essential amino acids, leading to an IGF1-deficient state, reduce age-dependent cognitive decline in an AD mouse model (Brandhorst et al., 2015). Periodic cycles of a fasting diet reduce hippocampal IGF-1 levels while increasing IGF-1 receptor mRNA expression and hippocampal neurogenesis (Brandhorst et al., 2015). Likewise, mice deficient in circulating IGF-1 due to either a GH or GHR deficiency display improved age-dependent cognitive performance compared with controls (Kinney-Forshee et al., 2004).

Past studies of intelligence in humans with GHRD have also been mixed. Some affected individuals show normal intelligence (Kranzler et al., 1998) and normal brain appearance, whereas others show varying degrees of parenchymal loss and below average intelligence (Shevah et al., 2005). These mixed findings may be explained partially by the different nature of specific GHR mutations, which may affect GHR and IGF-1 function differently during development, by variable degrees of inbreeding of the background populations, or by coexisting brain disease of miscellaneous etiologies.

GH- and GHR-deficient mice display record longevity extension for mice and a remarkably healthy phenotype throughout their lifespan (Bartke and Brown-Borg, 2004). Similarly, our previous results showed that, due to a splice site mutation at codon 180, exon 6 of the GHR (E180), humans develop GHRD and have a very low incidence of cancer and diabetes (Guevara-Aguirre et al., 2011). Here, we examined how this mutation affects brain structure and function. Participants were recruited from the Institute of Endocrinology, Metabolism, and Reproduction (IEMYR) of Quito, Ecuador, and brought to the University of Southern California (USC) in Los Angeles for cognitive testing. Thirteen young and middle-aged adults with GHRD and 12 of their relatives underwent MRI and cognitive assessment. Because of the known effects of IGF-1 on the cortex, myelin, and hippocampus, we included structural analyses focusing on these regions. Using fMRI, we evaluated hippocampal function while participants performed a pattern separation task known to rely on the dentate gyrus (DG) and CA3 of the hippocampus in older adults (Yassa et al., 2011).

Based on prior work in GHR knock-out mice (Ransome et al., 2004) and on our observations related to interventions that decrease IGF-1 in rodents (Brandhorst et al., 2015) and humans (Guevara-Aguirre et al., 2011, 2015), we tested the hypothesis that, compared with controls, individuals with GHRD may also have an imaging profile consistent with that of younger individuals. After the main analyses comparing the two groups, we further investigated whether our results were explained by variability in circulating levels of IGF-1 and IGFBP-1. IGFBP-1 is an IGF-binding protein that typically reduces IGF-1 action (Lee et al., 1997). IGFBP-1 expression is controlled mainly through in-
Table 1. Demographics of the participants

\begin{tabular}{lcc}
\hline & Control & GHRD \\
\hline No. of participants & 12 & 13 \\
Sex (M/F) & $3 / 9$ & $3 / 10$ \\
Age (mean \pm SD) & $40.6 \pm 8.9$ & $38.2 \pm 10.3$ \\
Age range & $24-54$ & $25-54$ \\
Education (years) & $10.8 \pm 4.6$ & $12.5 \pm 3.7$ \\
GHR E180 mutation & $11+/-; 1-/-$ & $13+/+$ \\
Plasma total IGF-1 (mean \pm SD) & $66.58 \pm 14.43$ & $15.15 \pm 6.24$ \\
Plasma free IGFBP-1 (mean \pm SD) & $2.82 \pm 2.52$ & $45.37 \pm 61.82$ \\
\hline
\end{tabular}

Age and years of education were not significantly different between diagnostic groups (two-tailed unpaired $t$ test; $p=0.53$ and 0.29 , respectively). Sex was not different between diagnostic groups $(c 2=0.01 ; p=0.91$ ).

sulin signaling. Lower insulin levels, as detected in GHRD subjects (Guevara-Aguirre et al., 2015), are associated with elevated IGFBP-1 expression (Clemmons, 1997).

\section{Materials and Methods}

\section{Study participants}

Participants were recruited and screened at the IEMYR and all neurological and cognitive testing was conducted at the USC Dana and David Dornsife Neuroimaging Center. Twenty-five participants (13 with GHRD and 12 unaffected relatives; age 24-54; mean 39.3 years; 6 males and 19 females; Table 1) were scanned. The GHRD participants were homozygous for an A-to-G splice site mutation at codon 180 in exon 6 of the growth hormone receptor (GHR) gene (E180 mutation). This mutation results in GHRD, characterized by very low $(\leq 20 \mathrm{ng} / \mathrm{ml})$ circulating IGF1 levels and is associated with severe short stature (Guevara-Aguirre et al., 2011). The controls were relatives of the GHRD participants with one wild-type and 11 heterozygous carriers for the E180 mutation. Participants were native Spanish speakers residing in Ecuador and all neuropsychological assessments were administered in Spanish. They signed written informed consent approved by the USC Institutional Review Board. One participant in the GHRD group was excluded from all analyses due to a brain structural abnormality. This participant had a Chiari type I hindbrain malformation with hydromyelia of the cervical spinal cord. Another GHRD participant was excluded from fMRI analyses due to scanner artifacts. Two GHRD participants were excluded from the pattern separation analyses because their tasks responses were not properly recorded.

\section{Assays and genotyping}

Plasma levels of total IGF-1 and free IGFBP-1 were measured. Total plasma IGF-1 was measured using an in-house kit developed by the Cohen laboratory using a double-monoclonal antibody ELISA with a sensitivity of $0.1 \mathrm{ng} / \mathrm{ml}$ and intra-assay and interassay coefficients of variation of $<6 \%$ and $<8 \%$, respectively, in the range of $1-6 \mathrm{ng} / \mathrm{ml}$ (Muzumdar et al., 2006). Free plasma IGFBP-1 was measured using a Quantikine ELISA kit (DGB100; R\&D Systems). Because of the positive skew to the IGFBP-1 and, to a lesser extent, IGF-1 values, a log transformation was performed on these values before including them in the statistical analyses. These log-transformed measures are indicated here by IGF- $1_{\log 10}$ and IGFBP- $1_{\log 10}$.

Saliva samples were collected with the Oragene OG-250 DNA collection kit (DNA Genotek) and processed according to manufacturer's protocol. Genotyping of the Ecuadorian splicing mutation (E180: A to G; Rosenfeld et al., 1994) was performed using PCR with following primers: $5^{\prime}$-cattgccctcaactggactt-3' (forward), 5'-cattttccatttagtttcatttact- $3^{\prime}$ (reverse, for WT), and $5^{\prime}$-cattttccatttagtttcatttacc- $3^{\prime}$ (reverse, for mutant) and cycling conditions of $95^{\circ} \mathrm{C}$ for $2 \mathrm{~h} ; 94^{\circ} \mathrm{C}$ for $45 \mathrm{~min}, 57.5^{\circ} \mathrm{C}$ for 45 $\min , 72^{\circ} \mathrm{C}$ for $45 \min (\times 35)$ and $72^{\circ} \mathrm{C}$ for $7 \mathrm{~h}$.

\section{MRI acquisition}

Imaging was conducted with a $3 \mathrm{~T}$ Siemens MAGNETOM Trio scanner with a 12-channel matrix head coil at the USC Dana and David Dornsife Neuroimaging Center. T1-weighted 3D structural MRI brain scans were acquired using a Magnetization Prepared Rapid Acquisition Gradient Echo (MPRAGE) sequence $(\mathrm{TR} / \mathrm{TE}=2530 / 3.13 \mathrm{~ms}$ FOV $=256 \times 256$ 


\section{Table 2. Freesurfer ROIs included in statistical analyses}

\begin{tabular}{l} 
Frontal cortex \\
Caudal middle frontal \\
Lateral orbitofrontal \\
Medial orbitofrontal \\
Rostral middle frontal \\
Superior frontal ${ }^{a}$ \\
Frontal pole $^{a}$ \\
Pars opercularis \\
Pars orbitalis \\
Pars triangularis \\
Cingulate cortex \\
Caudal anterior cingulate ${ }^{a}$ \\
Rostral anterior cingulate $^{a}$ \\
Temporal cortex \\
Entorhinal cortex $_{\text {Parahippocampal gyrus }}$ \\
Parietal cortex \\
Precuneus \\
\hline${ }^{a}$ Showed a significant difference between diagnostic groups in our fMRl analyses.
\end{tabular}

$\mathrm{mm}$; $256 \times 256 \mathrm{~mm}$ acquisition matrix; $1 \times 1 \times 1 \mathrm{~mm}^{3}$ voxels $/ 0 \mathrm{~mm}$ gap). To evaluate hippocampal subregions, a high-resolution T2weighted scan acquired perpendicular to the long axis of the hippocampus $(\mathrm{TR} / \mathrm{TE}=10,000 / 88 \mathrm{~ms} ;$ FOV $180 \times 180 \mathrm{~mm} ; 256 \times 256 \mathrm{~mm}$ acquisition matrix; $403 \mathrm{~mm}$ slices/ $0 \mathrm{~mm}$ gap; 3 averages; $0.7 \times 0.7 \times 3$ $\mathrm{mm}^{3}$ resolution) was used. Participants also underwent fMRI scanning $(\mathrm{TR} / \mathrm{TE}=2000 / 25 \mathrm{~ms} ; \mathrm{FOV}=192 \times 192 \mathrm{~mm} ; 64 \times 64 \mathrm{~mm}$ acquisition matrix; flip angle $(\mathrm{FA})=90^{\circ}$; Bandwidth $=2520 \mathrm{~Hz} / \mathrm{Px} ; 413 \mathrm{~mm}$ oblique axial slices; 210 images) and diffusion tensor imaging (DTI) scans $(\mathrm{TR} / \mathrm{TE}=8100 / 69 \mathrm{~ms}$; FOV $=256 \times 256 \mathrm{~mm}$; acquisition matrix $128 \times 128 \mathrm{~mm})$. Each participant's scan included $1 b_{0}$ and 64 diffusionweighted images $\left(b=1000 \mathrm{~s} / \mathrm{mm}^{2}\right)$.

\section{Structural analyses}

Cortical thickness, surface area, and hippocampal volume preprocessing. Cortical regions were selected a priori because they were hypothesized to be affected preferentially by IGF-1 and/or were identified as showing significant differences between diagnostic groups in fMRI analyses (Table 2). The T1-weighted structural images were used to calculate the bilateral mean cortical thickness, surface area, and hippocampal volume in these three main regions: frontal, medial temporal, and cingulate cortex (Table 2), which were selected for the following reasons. First, IGF-1 and IGFR-1 can be found in primate frontal cortex (Cheng et al., 2001; Xie et al., 2013). Second, physical activity promotes human brain regeneration, an effect that is dependent on IGF-1 (Carro et al., 2000; Trejo et al., 2001). These effects can be found in the hippocampus (Pajonk et al., 2010; Erickson et al., 2011), cingulate gyrus, and prefrontal cortex (PFC) (Colcombe et al., 2006; Flöel et al., 2010), suggesting the importance of IGF-1 signaling in these regions. Third, medial temporal cortex was selected for its proximity to the hippocampus and vulnerability in cognitive aging. Finally, because a significant group difference was found in the precuneus in the resting-state functional analysis, structural differences were also examined in this region. Right and left ROIs were examined separately only for those regions that showed significant mean bilateral group differences.

The whole-brain structural scans were processed as follows. First, a brain mask was created using the Analysis of Functional Neuroimages (AFNI) 3dSkullStrip tool (http://afni.nimh.nih.gov/afni/). The Advanced Neuroimaging Tools (ANTs) (http://stnava.github.io/ANTs/) $\mathrm{N} 4$ tool was used, with the brain mask generated in AFNI, and a b-spline distance of $100 \mathrm{~mm}$ to perform bias correction (Tustison et al., 2010). Next, the FreeSurfer image analysis suite (version 5.3.0; http://surfer. nmr.mgh.harvard.edu/) was used on the N4-corrected images to perform fully automated cortical reconstruction, GM segmentation, and calculation of cortical thickness as described previously (Dale et al., 1999; Fischl and Dale, 2000). FreeSurfer was also used to perform an initial segmentation of the hippocampus from surrounding tissue. A single researcher, blinded to all demographic and diagnostic information, re- viewed and manually edited the gray-white cortical boundaries and hippocampal segmentations as needed using TKMedit and FreeView software, respectively. Hippocampal segmentations were edited using a standardized segmentation protocol (Frisoni et al., 2015). FreeSurfer segmented the cortex into 34 regions in each brain hemisphere. Of these regions, statistical analyses were limited to 14 regions (Table 2) averaged across the right and left hemispheres to optimize statistical power.

To adjust for head size differences, each surface area region and hippocampal volume was divided by the intracranial volume (ICV). FreeSurfer version 5.3.0 was used to estimate intracranial volume as the template ICV multiplied by the inverse of the determinant of the transformation matrix that aligned individual T1-weighted scans to that template. To ensure that this estimation was not inappropriately influenced by smaller head size, the ICV values were compared with the volumes of visually inspected FreeSurfer skull-stripped brains. In these young and middle-aged adults, atrophy was minimal, so total brain size and ICV were expected to be closely related, which turned out to be the case ( $r=$ $\left.0.97 ; p=3.6 \times 10^{-15}\right)$. The difference between brain volume and ICV was not significantly correlated with diagnostic group $(r=0.10 ; p=$ 0.64 ), suggesting that the estimation of ICV was not affected by head size in a manner that would influence study results. There are multiple widely used ways to adjust for head size in studies of regional brain volume or surface area. Because of this, in addition to adjusting the volumes of the hippocampus and hippocampal subregions and selected cortical surface areas by intracranial volume in a proportional manner (i.e., dividing by ICV), analyses used raw volume and surface area measures and ICV was included as a covariate in multiple regression models.

Hippocampal subregion analyses. The hippocampal high-resolution T2-weighted scans were used to calculate the hippocampal subregion volumes for CA1, the DG, and a region that included CA2 and CA3 (CA23). Volumes were calculated using the Automatic Segmentation of Hippocampal Subfields (ASHS) tool. The mean of right and left for each hippocampal subregions were divided by the ICV for that participant to adjust for differences in head size.

DTI analyses. Fractional anisotropy (FA) and mean diffusivity (MD) were calculated. FA represents the degree to which water diffusion is directionally constrained. Lower FA, typically associated with neurological disease symptoms and slower nerve conduction (Thomason and Thompson, 2011), may indicate demyelination or less brain fiber coherence. $M D$ represents the mean of the eigenvalues along the three primary axes of water diffusion and tends to be higher in tissues that are less dense. Lower MD may reflect greater neural plasticity (Sagi et al., 2012). Both FA and MD may also be influenced by factors such as fiber coherence and fiber crossings, which do not necessarily reflect white matter integrity (Jones et al., 2013).

First, nonbrain matter was removed from the T1-weighted images using FreeSurfer (version 5.3.0) and the skull-stripped brains were linearly transformed ( 9 degrees of freedom; dof) to the Montreal Neurologic Institute (MNI) standard template (MNI152) space using FSL FLIRT (http://fsl.fmrib.ox.ac.uk/fsl/fslwiki/FLIRT). Next, FSL eddy correct (http://fsl.fmrib.ox.ac.uk/fsl/fslwiki/eddy) was used to correct for eddy current-induced distortions and participant movements in the diffusion scans. The eddy-corrected DTI scans were linearly transformed ( 9 dof) to MNI space and FSL's dtifit tool was used to calculate FA and MD.

To aid in aligning white matter tracts, a study-specific FA template was created based on all participants in the study. To do this, ANTs was used to create a minimal deformation template based on all T1-weighted scans (MDT-T1) in the study (Avants et al., 2010). The ANTs antsRegistration tool was used to transform each participant's T1-weighted scan nonlinearly to the MDT-T1. The antsApplyTransforms tool was then used to apply those nonlinear transformations to the FA and MD maps, which were already linearly aligned to the T1-weighted scans. This likewise transformed the FA and MD maps to MDT-T1 space. Next, ANTs was used to create a study-specific FA template (MDT-FA) based on the FA maps that had been transformed nonlinearly to MDT-T1 space. antsRegistration was used to transform each FA map nonlinearly in MDT-T1 space to the MDT-FA and those transformations were applied to the MD images to align them nonlinearly to the MDT-FA. 
A FreeSurfer-derived white matter segmentation mask was created for each participant. From this, a white matter template brain was created that included only voxels that are considered white matter in all participants. Statistics were evaluated only in those voxels.

FA and MD differences between the GHRD and control groups were assessed while adjusting for age, sex, and ICV (Takao et al., 2011).

Statistical analyses. ICV was compared in GHRD participants versus controls using multiple linear regression controlling for sex. Group differences in ICV-adjusted cortical surface area and cortical thickness in each of 14 mean bilateral FreeSurfer regions of interest (ROIs) were evaluated (Table 2). The significant correlations were further investigated by evaluating the relationship between the ICV-adjusted surface areas and circulating IGF- $1_{\log 10}$ and IGFBP- $1_{\log 10}$ levels while controlling for age, sex, and education. The same analyses were then run again, this time controlling for age, sex, education, and GHRD status (i.e., GHRD vs control) to evaluate what relationship, if any, IGF- $1_{\log 10}$ or IGFBP- $1_{\log 10}$ levels had to regional cortical surface area independent of GHRD status. An additional supplementary analysis of cortical surface area was also performed in the same 14 regions, but controlling for ICV as a regression covariate rather than dividing by it. Age, sex, and years of education were included as covariates in all analyses. ICV was not adjusted for when evaluating cortical thickness, which is thought to be independent of head size (Barnes et al., 2010). Within each analysis, the false discovery rate (FDR) was used to control for multiple comparisons when evaluating the relationship between mutation status and surface area across the ROIs that were evaluated (i.e., 14 comparisons). Using FDR, a result is considered significant if its $p$-value is below a critical $p$-value calculated to control false positives at the conventional rate of $<5 \%(q=0.05)$.

Surface area in a given region may appear greater because of different delineation of regions between groups. If this were the case, then GHRD might be associated with greater surface area in one region and with smaller surface area in the adjacent areas. To further evaluate our significant findings, this was tested by performing an additional surface area analysis of all 34 FreeSurfer cortical regions (bilateral means), again controlling for age, sex, and education.

In an exploratory analysis, differences in vertexwise cortical thickness between GHRD participants and controls were evaluated, controlling for age, sex, education, and ICV.

Using multiple linear regression, group differences in ICV-adjusted mean hippocampal volume and ICV-adjusted mean CA1, DG, and CA23 subregions were evaluated, covarying for age, sex, and education. A supplementary analysis of gross hippocampal and hippocampal subregional volumes was also performed, but controlling for ICV as a regression covariate rather than dividing by it.

Voxelwise DTI FA and MD differences between GHRD participants and controls were assessed, adjusting for age, sex, and ICV. Voxelwise multiple comparisons were corrected for (FDR $q=0.05)$. To ensure that the significant results did not relate to differences in education level, the same voxelwise analysis was run, this time controlling for age, sex, ICV, and years of education. The significant correlations were further investigated by evaluating the relationship between DTI voxelwise MD and IGF- $1_{\log 10}$ and IGCBP- $1_{\log 10}$ levels while controlling for age, sex, education, and ICV. The same analysis was run again, this time controlling for age, sex, education, ICV, and GHRD status to evaluate what relationship, if any, IGF-1 and IGFBP-1 levels had to DTI MD independent of GHRD status.

\section{fMRI preprocessing and analyses}

Behavioral task in the scanner. A modified version of the pattern separation task used by Yassa et al. (2011) was used (Fig. 1). Participants viewed a series of pictures in four blocks. In each block, 32 of 80 pictures appeared with a red border, indicating a new picture to be remembered for a later test. The rest of the 48 images were "test pictures" equally divided into three categories: 16 foils, 16 lures, and 16 repetitions. Foils were previously unviewed pictures that were not indicated as new. Lures were similar to a previously viewed picture. Repetitions were identical to a previously viewed picture. Participants pressed buttons to indicate whether each test picture was "new," "similar" to, or the "same" as a prior picture in the same block.
As in Yassa et al. (2011), a separation bias score was computed, which represents the likelihood that the item was correctly identified. This score was calculated as the proportion of lure items called similar minus the proportion of foils called similar ( $p$ [similar/lure $]-p$ [similar/foil $])$. The group difference in this score was evaluated using a univariate analysis while controlling for age, sex, and education.

Data preprocessing. FMRIB's Software Library (FSL; www.fmrib.ox.ac. uk/fsl) was used, which included motion correction with MCFLIRT, spatial smoothing with a Gaussian kernel of full-width half-maximum 5 $\mathrm{mm}$, high-pass temporal filtering equivalent to $100 \mathrm{~s}$, and skull stripping of structural images with BET. Registration was performed with FLIRT; each functional image was registered to both the participant's high-resolution brain-extracted structural image and the study specific template.

Whole-brain analysis. Whole-brain analyses were conducted using FSL FEAT version 6.00. For each run for each participant, stimulusdependent changes in BOLD signal were modeled with six regressors based on trial type and behavioral responses. The first four regressors were correct responses, including, lures called similar ("similar"), repetitions called same ("same"), foils called new ("new"), and red borders called new ("border"). The border was a regressor of no interest. As in previous studies, "new" served as baseline. The next two regressors were error trials; lures called same (error-lure) and the rest of the error trials of no interest (error -rest $_{\text {) }}$. Error_lure was separately modeled from the rest of the errors due to the fact that previous studies have shown aging effects in the similar $>$ error-lure contrast (Yassa et al., 2011).

For each participant, brain activity differences were examined for the following: (1) similar > baseline, (2) same > baseline, and (3) similar > error $_{\text {lure }}$. The regressors were convolved with a double-gamma hemodynamic response function and its temporal derivative with the six head movement parameters as nuisance variables. Temporal filtering was also applied to the model. The resulting images were entered into the secondlevel analysis to estimate average activation across four blocks for each participant. At the third-level (group level), group differences in brain activation were evaluated in each of the contrasts above while controlling for age, sex, education, and ICV. $Z$ (Gaussianized T/F) statistic images were thresholded at the whole-brain level using clusters determined by $Z>2.3$ and a corrected-cluster significance threshold of $p=0.05$ (Worsley, 2001). The relationship between brain activation and circulating IGF- $1_{\log 10}$ and between brain activation and IGFBP- $1_{\log 10}$ levels were also examined while controlling for age, sex, education, and ICV using the same threshold.

ROI analyses. ROI analyses were conducted for the left and right hippocampi. The masks were created by transforming the Harvard-Oxford defined hippocampi to our study template using ANTs and FSL FLIRT. ROI analyses for the hippocampal subfields (CA23/DG and CA1) were also performed, given their key roles in this task (Guzowski et al., 2004; Bakker et al., 2008). Using univariate analyses, group differences in mean percentage signal change were evaluated for each region. The masks were created for each participant based on our hippocampal high-resolution scans (see "Hippocampal subregion analyses" section) and were transformed to fMRI space using FSL FLIRT. In an exploratory analysis, multiple linear regression analyses were performed to examine the relationship between IGF- $1_{\log 10}$ levels and brain activation in the regions showing a significant group difference. The same analyses were performed using IGFBP- $1_{\log 10}$ levels.

Brain-behavior relationships. Multiple linear regression analyses were performed to test the association between behavioral performance (separation bias score) and fMRI activity (regional mean percentage signal change) in the each region that showed a significant group difference.

Resting-state analyses. The data were preprocessed in the same way as the task fMRI data. To examine group differences in functional connectivity for the default mode network, seed ROIs were selected a priori, namely the bilateral posterior cingulate cortex/precuneus and the left and right hippocampi. The PCC was defined based on previous research (Hedden et al., 2009): a 4-mm-radius sphere in the PCC (MNI coordinates: $0,53,26)$. The subject-specific hippocampal masks that were already segmented were used with FreeSurfer (see "Cortical thickness, surface area, and hippocampal volume preprocessing" section). Each subject's hippocampi were transformed into fMRI space using 


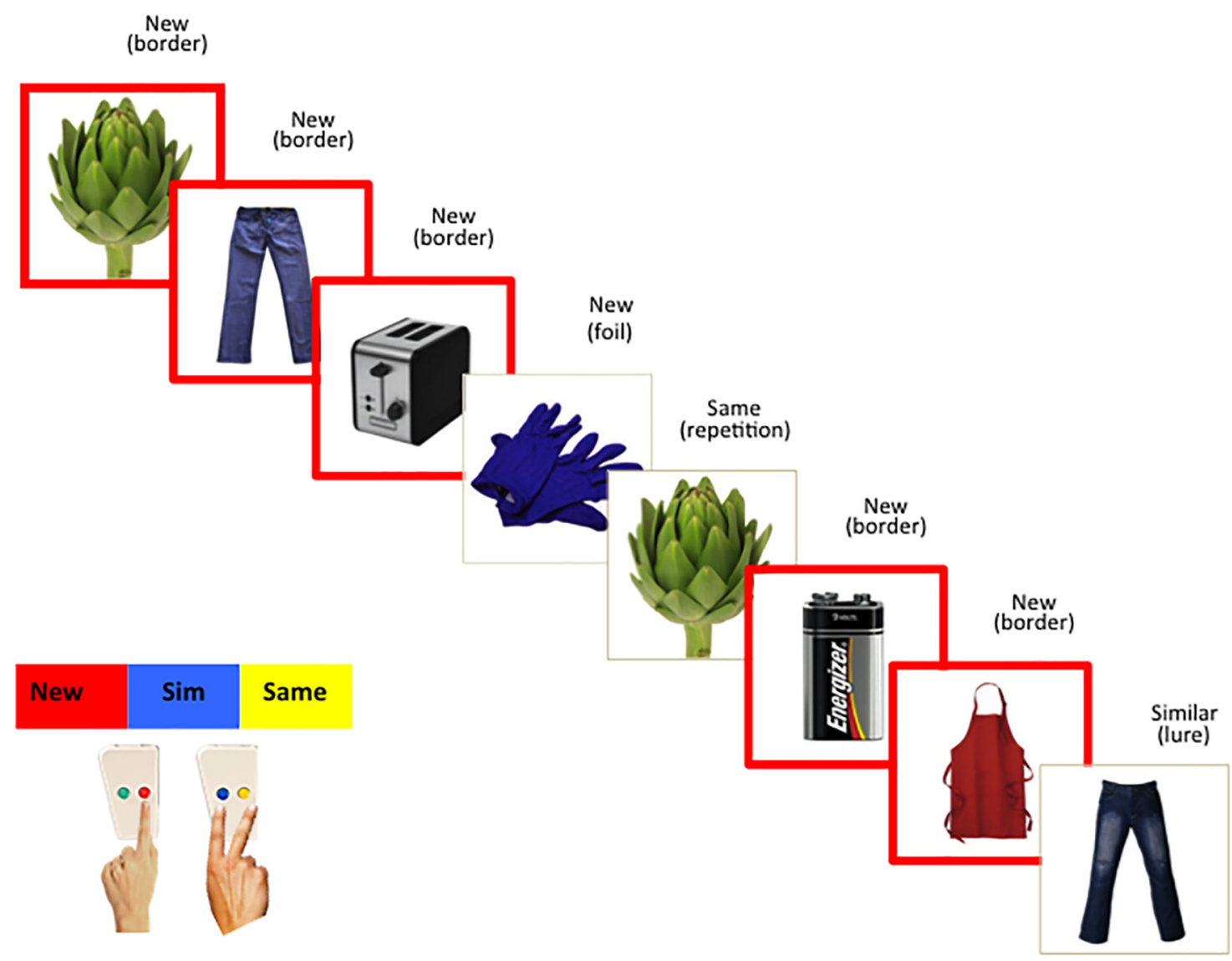

Figure 1. Pattern separation task. Stimuli were color photographs of common objects and scenes from the International Affective Picture System (Lang et al., 2005). The images with a red border indicate that they were presented for the first time and that participants' memory for these items would be tested later. Participants then viewed one of the three types of images: foils, lures, or repetitions. Foils had not been presented before. Lures were similar to those seen before. Repetitions had been seen previously. Participants were asked to respond to each picture by indicating whether the picture was new, similar, or the same by using a button press (new = red; similar = blue; same = yellow; the green button was unused). In the above figure, correct responses were displayed on top of each picture and types of images were shown in parentheses, which were not shown to the participants. Behavioral performance (separation bias score) and brain activation for nonbordered items were used for analyses. Participants completed four blocks, each with a randomized presentation of 32 red-bordered pictures, 16 foils, 16 lures, and 16 repetitions. Each stimulus was presented for $3000 \mathrm{~ms}$, followed by an interstimulus interval fixation cross of $500 \mathrm{~ms}$. We calculated response rates in each of the nine possible categories (foils called same, similar, or new; lures called same, similar, or new; and repetitions called same, similar, or new).

mri_label2vol and flirt. For each seed ROI, the mean resting-state BOLD time series was extracted for each participant. The mean time series from each ROI was included in a general linear model, along with nine regressors of no interest, including six motion parameters, the time series of the white matter, the CSF, and the whole brain. Group differences in functional connectivity were then evaluated using clusters determined by $Z>$ 2.3 and a corrected-cluster significance threshold of $p=0.05$. In addition, the relationship between IGF- $1_{\log 10}$ levels and functional connectivity with each seed region were examined using the same threshold. The same analyses were performed using IGFBP- $1_{\log 10}$ levels.

Statistical functional analyses. Age, sex, education, and ICV were controlled for in all fMRI analyses.

\section{Results}

\section{Structural analyses}

Cortical surface area and thickness

As expected, ICV was significantly smaller in GHRD participants versus controls after controlling for sex $\left(R^{2}[\right.$ full model $]=0.58$; $p[$ full model $]=0.0001 ; p[$ partial contribution of $\mathrm{GHR}]=$ $0.00005)$.

After controlling for age, sex, and education, the ICV-adjusted regional surface area was significantly larger (FDR critical $p=$ $0.014)$ in GHRD participants in the frontal pole $(p=0.002)$ and in the caudal middle frontal $(p=0.005)$, medial orbitofrontal $(p=0.0006)$, rostral middle frontal $(p=0.006)$, superior frontal $(p=0.014)$, and rostral anterior cingulate $(p=0.010)$ cortices.

When IGF- $1_{\log 10}$ was used as the independent variable rather than GHRD status (controlling for age, sex, and education), higher IGF- $1_{\log 10}$ levels were significantly associated with smaller ICV-adjusted surface area in all of these same regions (except rostral middle frontal $p=0.05$ ) and additionally in the lateral orbitofrontal region (FDR critical $p=0.0128$; frontal pole $p=$ 0.00001 ; caudal middle frontal $p=0.0007$; medial orbitofrontal $p=0.00007$; superior frontal $p=0.0007$; and rostral anterior cingulate $p=0.0015$; lateral orbitofrontal $p=0.013$ ).

When IGFBP- $1_{\log 10}$ was used as the independent variable rather than GHRD status (controlling for age, sex, and education), higher IGFBP- $1_{\log 10}$ levels were significantly associated with larger ICV-adjusted surface area in all of the same regions as those associated with IGF-1, but with additional associations in the caudal anterior cingulate and parahippocampal cortex (FDR critical $p=0.0275$; frontal pole $p=0.00094$; caudal middle frontal $p=0.00026$; medial orbitofrontal $p=0.00024$; superior frontal $p=0.00012$; and rostral anterior cingulate $p=0.00007$; lateral orbitofrontal $p=0.010$; caudal anterior cingulate $p=0.011$; parahippocampal cortex $p=0.0275$ ). 
We also examined free IGFBP-1, that is, IGFBP-1 that is not bound to IGF-1. To some extent, levels of IGFBP-1 that remain free will depend on the amount of IGF-1 available for binding and the effect of IGF-1 will depend on the extent to which IGF-1 is inhibited by IGFBPs. We evaluated the effect of IGFBP- $1_{\log 10}$ on surface area while controlling for IGF- $1_{\log 10}$ and the effect of IGF- $1_{\log 10}$ on surface area while controlling for IGFBP- $1_{\log 10}$. We controlled for age, sex, and education in both analyses and found no significant effect on surface area of IGF-1 independent of IGFBP-1 or of IGFBP-1 independent of IGF-1.

Next, we assessed whether plasma IGF- $1_{\log 10}$ or IGFBP- $1_{\log 10}$ had a significant effect on surface area independent of GHRD status. We controlled for GHRD status in each analysis, along with age, sex, and education. We found that higher IGF- $1_{\log 10}$ was significantly associated with smaller surface area in the frontal pole independent of GHRD status ( $p=0.00076$; FDR critical $p=$ $0.00076)$. Higher IGFBP- $1_{\log 10}$ was significantly associated with larger surface area in the rostral anterior cingulate $(p=0.0031)$, superior frontal cortex $(p=0.0037)$, and pars opercularis $(p=$ 0.0027 ) independent of GHRD status (FDR critical $p=0.0037)$.

For the regions in which we found a significant effect of GHRD status, we then evaluated GHRD status group differences separately in the right and left hemispheres, controlling for age, sex, and education. The surface area was significantly (FDR critical $p=0.017$ ) greater in GHRD versus control in the right and left hemispheres, respectively, in the caudal middle frontal ( $p=$ 0.012 and 0.010$)$, medial orbitofrontal ( $p=0.010$ and 0.0002 ), and rostral middle frontal $(p=0.008$ and 0.017$)$ cortices. The surface area of the rostral anterior cingulate $(p=0.007)$ and superior frontal cortex $(p=0.004)$ was significantly greater in GHRD participants in the left hemisphere only and the surface area for the frontal pole $(p=0.0006)$ was significantly larger in GHRD participants on the right only. There were no significant group differences in cortical thickness in any of the regions evaluated or in a supplementary vertexwise analysis.

We then performed a supplementary analysis in all 14 cortical ROIs in which we evaluated the relationship between GHRD mutation and regional surface area, this time including ICV as a covariate, along with age, sex, and education rather than dividing by ICV. Using this alternative analysis, surface area in the mean bilateral rostral middle frontal cortex was still larger in GHRD mutation carriers compared with controls $(p=0.001$; FDR critical $p=0.001)$. Mean bilateral caudal middle frontal cortex showed a trend toward being larger in GHRD mutation carriers $(p=0.063)$.

If the larger surface areas that we found in GHRD participants in select areas were due to different automated delineation of cortical boundaries between groups, then surface areas in adjacent areas may conversely appear larger in controls. To determine whether greater surface area in the GHRD participants was accompanied by smaller surface area in the adjacent regions, we evaluated differences between GHRD participants and controls in all 34 FreeSurfer cortical regions (bilateral means). After controlling for age, sex, and education, ICV-adjusted bilateral regional surface area was larger in GHRD subjects for all regions that showed a significant difference (Table 3 ). These results suggest that our differences may be related to increased gyrification in GHRD subjects rather than to differences in cortical region delineation.

Another potential explanation for our results is that they are simply due to the different cortical geometry of smaller brains. However, in the general population, cortical surface area increases more with ICV than would be expected by a simple scaling
Table 3. Group differences in ICV-adjusted regional surface area

\begin{tabular}{ll}
\hline & $p$-value \\
\hline Frontal cortex & \\
Caudal middle frontal & 0.0045 \\
Lateral orbitofrontal & NS \\
Medial orbitofrontal & 0.00060 \\
Pars opercularis & NS \\
Pars orbitalis & NS \\
Pars triangularis & NS \\
Precentral gyrus & NS \\
Rostral middle frontal & 0.0060 \\
Superior frontal & NS \\
Frontal pole & 0.0015 \\
Parietal cortex & \\
Inferior parietal & NS \\
Pericalcarine cortex & NS \\
Postcentral gyrus & NS \\
Precuneus & NS \\
Superior parietal & NS \\
Supramarginal gyrus & NS \\
Frontal/parietal cortex & \\
Paracentral lobule & NS \\
Temporal cortex & \\
Banks of the superior temporal sulcus & NS \\
Entorhinal & NS \\
Fusiform & NS \\
Inferior temporal & NS \\
Middle temporal gyrus & 0.0049 \\
Parahippocampal gyrus & NS \\
Superior temporal & 0.011 \\
Temporal pole & NS \\
Transverse temporal gyrus & NS \\
Occipital cortex & \\
Cuneus & NS \\
Lateral occipital & 0.000098 \\
Lingual gyrus & NS \\
Cingulate cortex & \\
Caudal anterior cingulate & NS \\
Isthmus of the cingulate & NS \\
Rostral anterior cingulate & 0.0096 \\
Posterior cingulate & NS \\
Insular cortex & NS \\
\hline Int & \\
\hline
\end{tabular}

NS, Not significant.

factor (Im et al., 2008). Therefore, in the normal population, one would expect smaller brains to have less surface area after adjusting for ICV-the opposite of what we found. This suggests that greater gyrification is a more likely explanation for our results.

\section{Hippocampal volume}

ICV-adjusted volume of the DG $\left(R^{2}[\right.$ full model $]=0.30 ; p[$ full model $]=0.11 ; p[$ partial contribution of GHRD $]=0.05)$ and CA1 of the hippocampus $\left(R^{2}\right.$ [full model $]=0.38 ; p[$ full model $]=$ 0.04; $p$ [partial contribution of GHR] $=0.08$ ) showed a weak trend toward being larger in GHRD participants versus controls, covarying for age, sex, and education. The ICV-adjusted volume of CA23 was not significantly associated with GHRD status $\left(R^{2}[\right.$ full model $]=0.33 ; p[$ full model $]=0.07 ; p[$ partial contribution of GHRD] $=0.25$ ).

The ICV-adjusted mean hippocampal volume was not significantly associated with GHRD status after controlling for age, sex, and education $\left(R^{2}[\right.$ full model $]=0.28 ; p[$ full model $\left.]=0.14\right)$. In a supplementary analysis, we included ICV as a covariate rather than dividing hippocampal volume and subregional volumes by it. Using this method of adjusting for ICV, mean bilateral hip- 


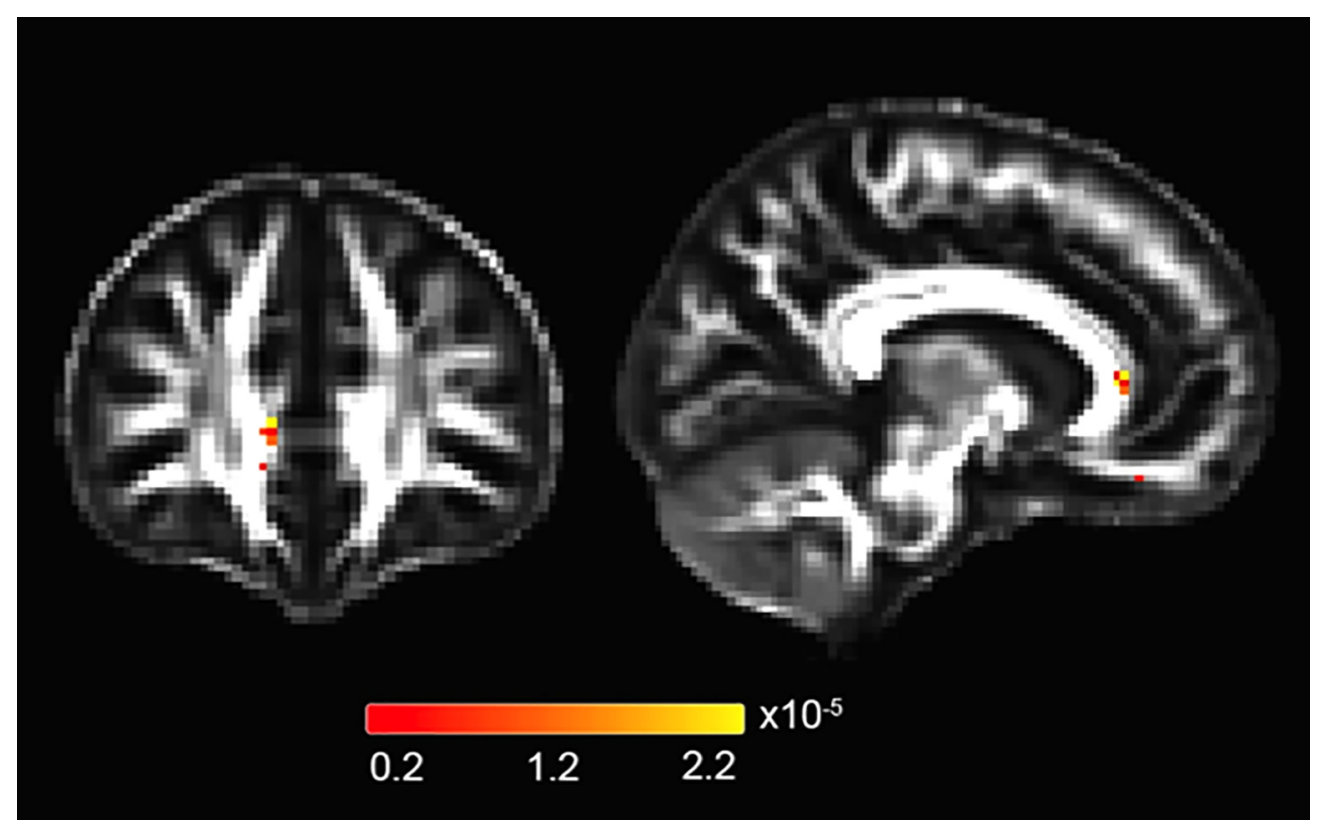

Figure 2. Group differences in DTI mean diffusivity. GHRD participants had lower DTIMD in the genu of the corpus callosum after controlling for age, sex, and ICV (critical $p$-value that controls FDR at $q=0.05$ is $2.35 \times 10^{-5}$ ). Left side of the brain is displayed on the right.

pocampus and hippocampal subregions still did not show a significant association with GHRD mutation.

\section{DTI}

After controlling for age, sex, and ICV, DTI MD was significantly lower in GHRD participants than controls, possibly indicating greater neural plasticity (Deijen et al., 2011). Regions of significance included the genu of the corpus callosum, the posterior limb of the internal capsule/anterior thalamic tracts, and the bilateral superior cerebellar peduncles/anterior thalamic tracts (critical $p=2.35 \times 10^{-5}$ controls FDR at $q=0.05$; Fig. 2 ). When this analysis was repeated by additionally controlling for education, MD was still significantly lower in GHRD participants in these regions ( critical $p=1.36 \times 10^{-5}$ controls FDR at $q=0.05$ ). We next used circulating IGF $1_{\log 10}$ as the independent variable rather than GHRD status, controlling for age, sex, ICV, and education. The same regions remain significantly correlated with DTI MD (critical $p=7.68 \times 10^{-6}$ controls FDR at $\left.q=0.05\right)$. However, these regions did not remain significantly correlated with MD when IGF $1_{\log 10}$ is the independent variable and GHRD status is also controlled for along with age, sex, ICV, and education. This suggests that either our study is not sufficiently powered to detect a IGF-1 effect that is independent of GHRD status or that an independent effect of IGF-1 on DTI MD does not exist in our participants. Plasma free IGFBP- $1_{\log 10}$ was not significantly associated with DTI MD after controlling for age, sex, ICV, and education.

After controlling for age, sex, and ICV, DTI FA was significantly higher, typically indicating more intact white matter, in GHRD participants versus controls in one isolated voxel in the left corticospinal tract (critical $p=4.03 \times 10^{-7}$ controls FDR at $q=0.05)$.

\section{FMRI analyses}

\section{Behavioral analysis}

The GHRD group showed a significantly higher pattern separation score than the control group $\left(F_{(1,18)}=11.78, \mathrm{MSE}=0.02\right.$, $p=0.003, \eta_{\mathrm{p}}^{2}=0.40$; Fig. 3$)$. GHRD participants correctly iden- tified the lure items as similar more frequently than did the control group $\left(F_{(1,18)}=8.44, \operatorname{MSE}=0.02, p=0.009, \eta_{p}^{2}=0.32\right)$. Relative to the GHRD group, the control group was more likely to label the lure items as same, indicating a higher rate of false alarms to lures $\left(F_{(1,18)}=4.72, \mathrm{MSE}=0.03, p=0.043, \eta_{\mathrm{p}}^{2}=0.21\right)$.

\section{Whole-brain analysis}

Compared with controls, the GHRD group showed greater activation in the bilateral superior frontal gyrus (SFG) in the similar $>$ baseline contrast (Fig. 4A, Table 4). GHDR participants also showed greater activation in the left superior parietal lobe (SPL) in the same $>$ baseline contrast (Fig. $4 B$, Table 4) and greater activation in the left frontal pole and in the bilateral anterior cingulate cortex (ACC) in the similar $>$ error $_{\text {lure }}$ contrast (Fig. 4C,D, Table 4).

When the IGF- $1_{\log 10}$ level was used as the independent variable rather than GHRD status, there were no significant findings. When IGFBP- $1_{\log 10}$ level was used as the independent variable rather than GHRD status, we found a negative relationships between IGFBP- $1_{\log 10}$ and several brain regions. Higher IGFBP$1_{\log 10}$ was associated with lower activation in the caudate, the right postcentral gyrus, the left occipital cortex, and frontal pole in the similar $>$ baseline contrast. For the similar $>$ error $_{\text {lure }}$ contrast, higher IGFBP-1 $1_{\log 10}$ was associated with lower activation in the left middle frontal gyrus, the bilateral occipital lobe, and the SPL.

\section{ROI analyses for the hippocampi}

Compared with controls, the GHRD group showed greater activation in the right hippocampus in the similar $>$ baseline contrast $\left(M_{\mathrm{GHRD}}=0.04 ; M_{\text {control }}=-0.06\right), F_{(1,16)}=5.78$, $\mathrm{MSE}=0.004, p=0.029, \eta_{\mathrm{p}}^{2}=0.26$; Fig. $\left.4 E\right)$. Significant group differences were not observed in the left hippocampus $(p=0.12)$. There were no significant findings when $I G F 1_{\log 10}$ or IGFBP$1_{\log 10}$ was used as the independent variable rather than GHRD status. 
A GHRD

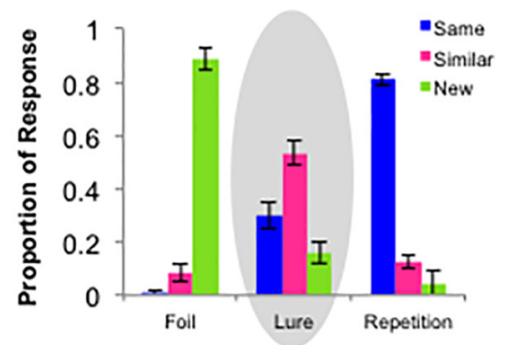

Trial Type
B Control

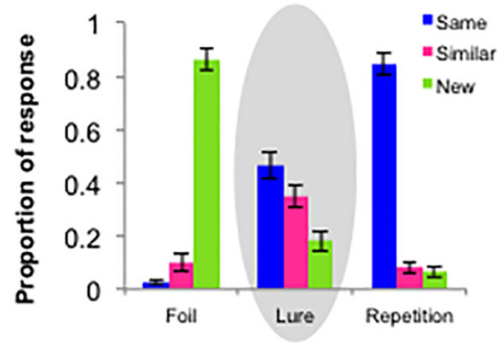

Trail Type

\section{Separation Bias}
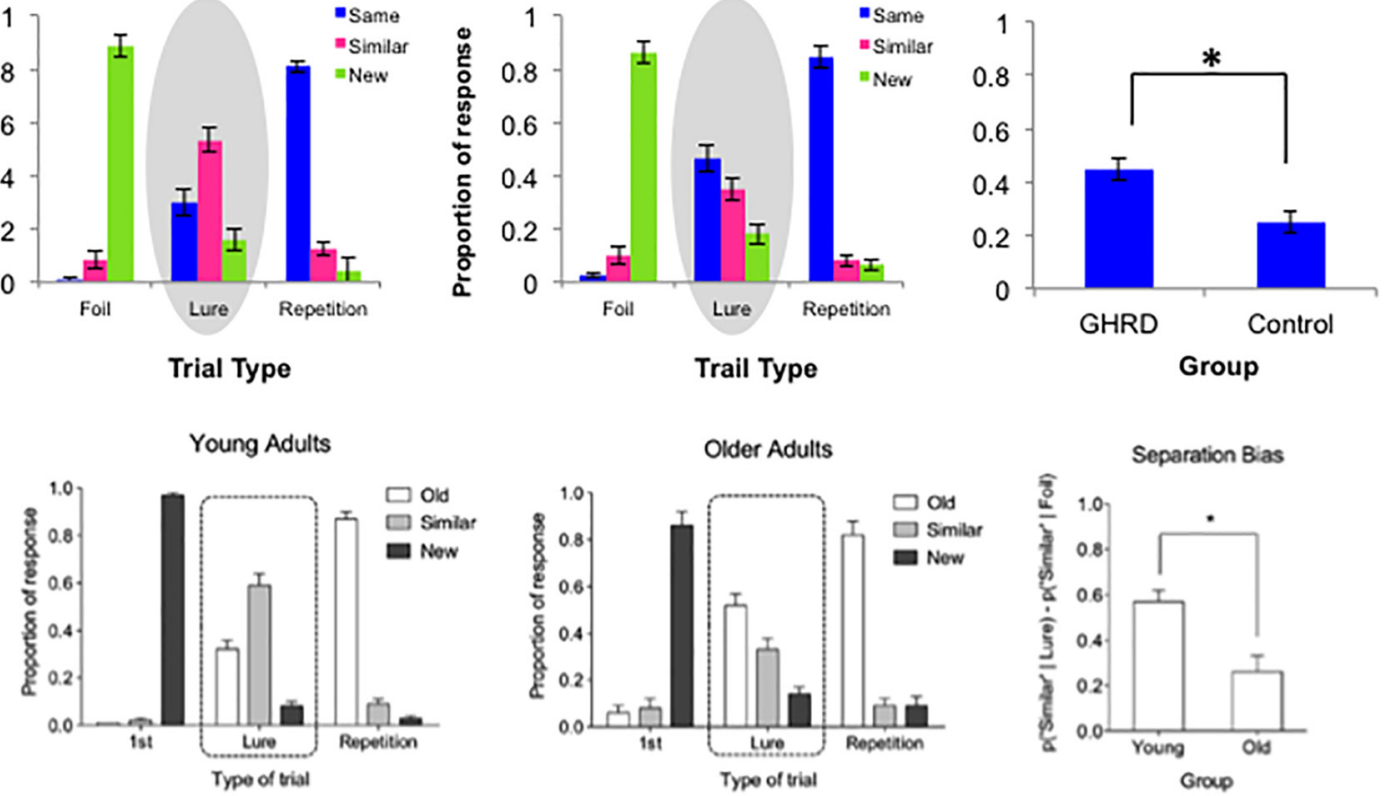

Figure 3. Group differences in separation bias scores. $\boldsymbol{A}, \boldsymbol{B}$, Response proportions displayed in the top graphs were adjusted for age, sex, and education. C, The GHRD group had greater separation bias scores than the control group after controlling for age, sex, and education, indicating better memory performance in the GHRD group. This pattern of group difference was similar to that observed in younger versus older adults in a previous study, as shown in the bottom graphs (adapted from Yassa et al., 2011). In this previous study, the average age of the younger adults was 20 years and that of older adults was 70 years. Conversely, our participants were young to middle-aged (range 24-54; Table 1). Because our control participants were, on average, much older (mean $=$ 40.6) than the younger participants in the previous study (mean $=20$ ), it is possible that our control group already showed signs of cognitive aging, whereas the GHRD group did not.

ROI analyses for the hippocampal subfields

Although the GHRD participants showed greater activation than controls in all subfields, the results did not reach significance $(p>0.12$; Fig. 5).

\section{Brain-behavior relationships}

Across groups, a higher separation bias score (better performance) was significantly associated with greater fMRI activity in the bilateral SFG $\left(R^{2}\right.$ [full model $]=0.74 ; p[$ full model $]<0.001$; $p$ [partial contribution of SFG activation] $=0.002$; Fig. $4 A)$, left SPL $\left(R^{2}\right.$ [full model $]=0.74 ; p[$ full model $]<0.001 ; p$ [partial contribution of SPL activation] $=0.002$; Fig. $4 B)$, left frontal pole (score $\left(R^{2}\right.$ [full model $]=0.73 ; p[$ full model $]<0.001 ; p[$ partial contribution of frontal pole activation] $=0.002$; Fig. $4 C)$, and bilateral ACC $\left(\right.$ score $\left(R^{2}\right.$ [full model $]=0.68 ; p$ full model $]=$ 0.002; $p$ [partial contribution of ACC activation] $=0.010$; Fig. $4 D)$. Greater activation in the right hippocampus was marginally significantly associated with higher separation bias score $\left(R^{2}\right.$ [full model $]=0.61 ; p[$ full model $]=0.006 ; p[$ partial contribution of hippocampal activation]0.052; Fig. $4 E$ ). When the analyses were repeated for each group separately, the results did not reach significance. These results suggest a more effective recruitment of cognitive resources in the GHRD participants.

In the brain regions showing inverse relationships with IGFBP1 levels, reduced activation was not significantly associated with better performance.

\section{Resting-state analyses}

Resting-state connectivity in the default mode network (DMN) typically decreases in AD and aging (Greicius et al., 2004; Mevel et al., 2013). Relative to controls, the GHRD group showed greater DMN functional connectivity in the precuneus extended to the left occipital cortex (Fig. 6). Similarly, the GHRD group showed greater DMN functional connectivity in the precuneus with the left and right hippocampal seed regions (Fig. 6). When IGF- $1_{\log 10}$ and IGFBP- $1_{\log 10}$ were used as the independent variable rather than GHRD status, there were no significant findings.

\section{Discussion}

Our findings suggest that the GHRD protective effects found in mice with decreased IGF-1 levels and enhanced insulin efficiency may also extend to the human brain. GHRD participants had greater regional cortical surface area relative to intracranial volume compared with controls in several frontal and anterior cingulate regions, including significant or trend-level effects in rostal and caudal middle frontal cortex, regardless of method of controlling for ICV. These results may indicate greater gyrification in subjects with GHRD. Circulating IGF-1 or IGFBP-1 levels may partly explain these effects. In addition, higher IGF-1 was associated with smaller surface area in the frontal pole and higher IGFBP-1 was associated with larger surface area in rostral anterior cingulate, superior frontal cortex, and pars opercularis independently of GHRD status. Because cortical gyrification primarily takes place prenatally (Armstrong et al., 1995), these effects may be developmental rather than aging related. We found no significant differences in cortical thickness between groups either within our ROIs or in a post hoc exploratory whole-brain vertexwise analysis. Possible explanations for this may be that the subjects in our sample were not old enough to have detectable age-related cortical thinning or the relatively small, crosssectional sample. Alternatively, it is possible that the GHRD mutation does not modulate the cortical thinning sometimes associated with aging. A larger longitudinal study that includes older subjects may help to elucidate this question.

We also saw trends toward larger ICV-adjusted DG and CA1 regions in the hippocampus. Furthermore, GHRD participants had lower mean diffusivity of white matter (possibly indicating 

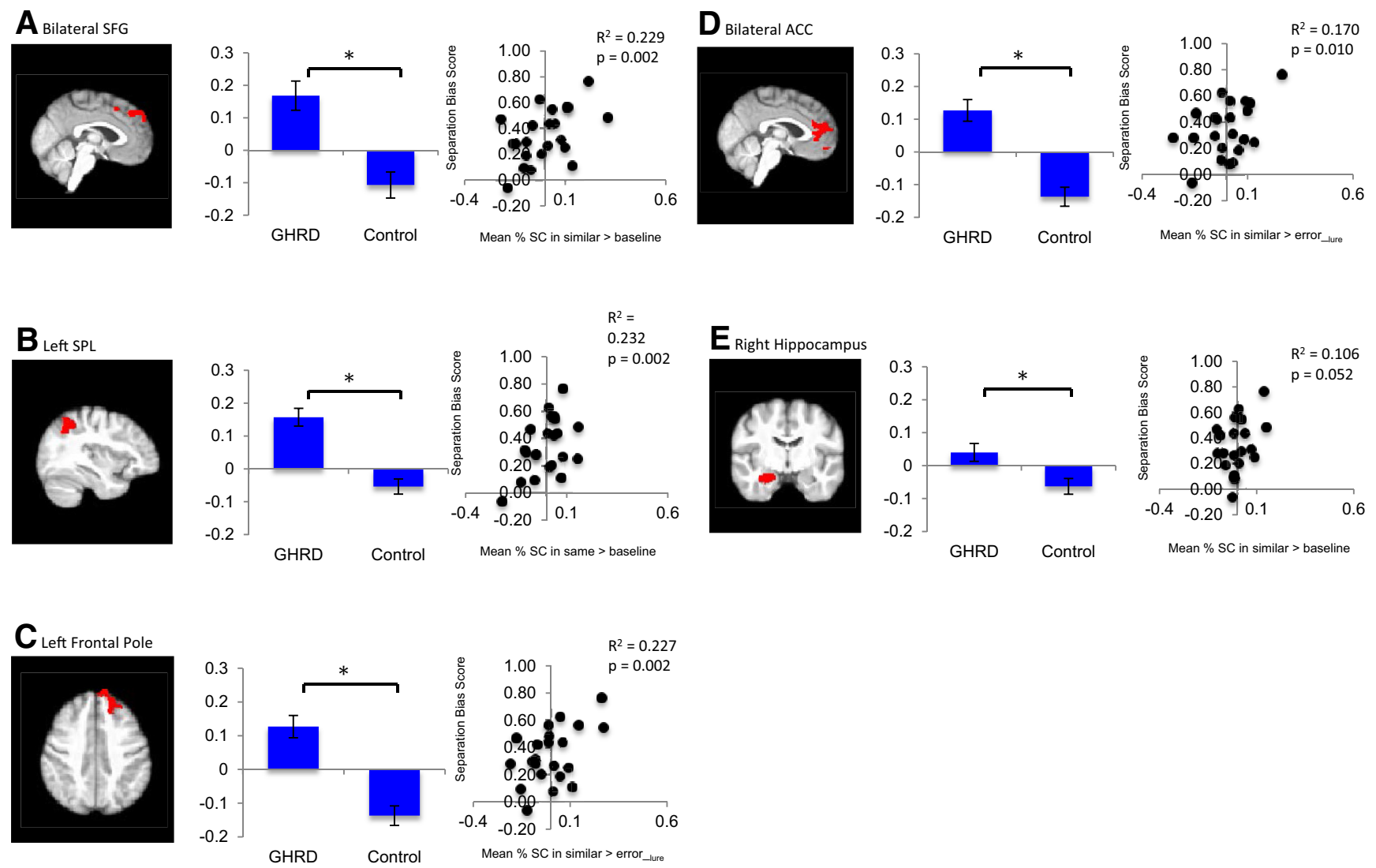

Figure 4. Brain regions showing greater activation in the GHRD group than controls during the pattern separation task. A, Compared with the control group, the GHRD group showed increased activation in the bilateral superior frontal gyrus during the similar trials relative to baseline. $\boldsymbol{B}$, The GHRD group also exhibited greater activation in the left superior parietal lobe during the same trials relative to baseline). C, D, Furthermore, the GHRD group showed greater activation in the left frontal pole $(\boldsymbol{C})$ and in the bilateral anterior cingulate cortex (D) in the similar $>$ error lure contrast. $\boldsymbol{E}$, The ROI analysis showed greater activation in the right hippocampus in the GHRD versus control groups in the similar $>$ baseline contrast. The bar graphs show mean percentage signal change in each ROI and the scatter plots depict the brain-behavior relationships in each ROI across groups. The mean percentage signal change represents residual values after controlling for age, sex, education, and ICV. The separation bias score was calculated by [ $p$ (similar/lure) $-p$ (similar/foil)]. Greater activation in these regions was associated with better separation bias score. $R^{2}$ and $P$ are the partial contribution of activation in each ROl to the full model.

Table 4. Brain activity showing significant group differences during pattern separation task

\begin{tabular}{|c|c|c|c|c|c|c|}
\hline \multirow[b]{2}{*}{ Contrast } & \multirow[b]{2}{*}{ Brain region } & \multirow[b]{2}{*}{$\mathrm{H}$} & \multicolumn{3}{|l|}{$\mathrm{MNI}$} & \multirow[b]{2}{*}{ Z-max } \\
\hline & & & $x$ & $y$ & $\bar{z}$ & \\
\hline \multicolumn{7}{|l|}{ GHRD $>$ control } \\
\hline Similar $>$ baseline & Superior frontal gyrus & $\mathrm{R}$ & 4 & 48 & 46 & 3.27 \\
\hline Same $>$ baseline & Superior parietal lobe & L & -36 & -54 & 52 & 3.68 \\
\hline \multirow[t]{2}{*}{ Similar > error_lure } & Anterior cingulate gyrus & $\mathrm{L}$ & -2 & 38 & 20 & 3.47 \\
\hline & Frontal pole & $\mathrm{L}$ & -20 & 42 & 42 & 3.55 \\
\hline Error_lure $>$ baseline & No significant results & & & & & \\
\hline \multicolumn{7}{|l|}{ Control $>$ GHRD } \\
\hline Similar $>$ baseline & No significant results & & & & & \\
\hline Same $>$ baseline & No significant results & & & & & \\
\hline Similar $>$ error_lure & No significant results & & & & & \\
\hline Error_lure $>$ baseline & No significant results & & & & & \\
\hline
\end{tabular}

Baseline indicates new trials.

greater myelination, tract coherence, or neural plasticity) in two tracts adjacent to the frontal and cingulate cortex: the genu of the corpus callosum and anterior thalamic tracts. This effect may be explained by IGF-1 levels.

Although the average age of the subjects tested was 39, in the pattern separation task, the GHRD group performed in the range expected for even younger adults (Fig. 3; Yassa et al., 2011). In addition, GHRD participants showed greater fMRI activation in frontoparietal regions and in the right hippocampus. Such in- creased activation was associated with better memory performance. Although we observed inverse relationships between IGFBP-1 levels and brain activations in some regions, reduced activations in these regions were not associated with better performance. Therefore, the effects of IGFBP-1 on cognitive function remain to be investigated.

Furthermore, our resting-state analyses indicated that the GHRD group has greater functional synchronicity of activity between the precuneus and seed regions in the PCC and in the left and right hippocampi. The precuneus is part of the DMN, which is the most commonly observed resting-state network associated with cognitive function. Given that the DMN is known to show age- and disease-related declines in functional synchronicity (Greicius et al., 2004; Mevel et al., 2013), the greater functional connectivity observed in the GHRD group may indicate healthier brain function. However, this interpretation needs to be made cautiously because increases in DMN functional connectivity have also been observed in pathological conditions such as in $A P O E \varepsilon 4$ carriers and people with depression (Greicius et al., 2007; Westlye et al., 2011). To explore this question further, we examined the relationship between resting-state functional synchronicity and performance in the pattern separation task using multiple linear regression. The results suggested that, across groups, greater functional synchronicity between the precuneus and the right hippocampus was associated with better perfor- 

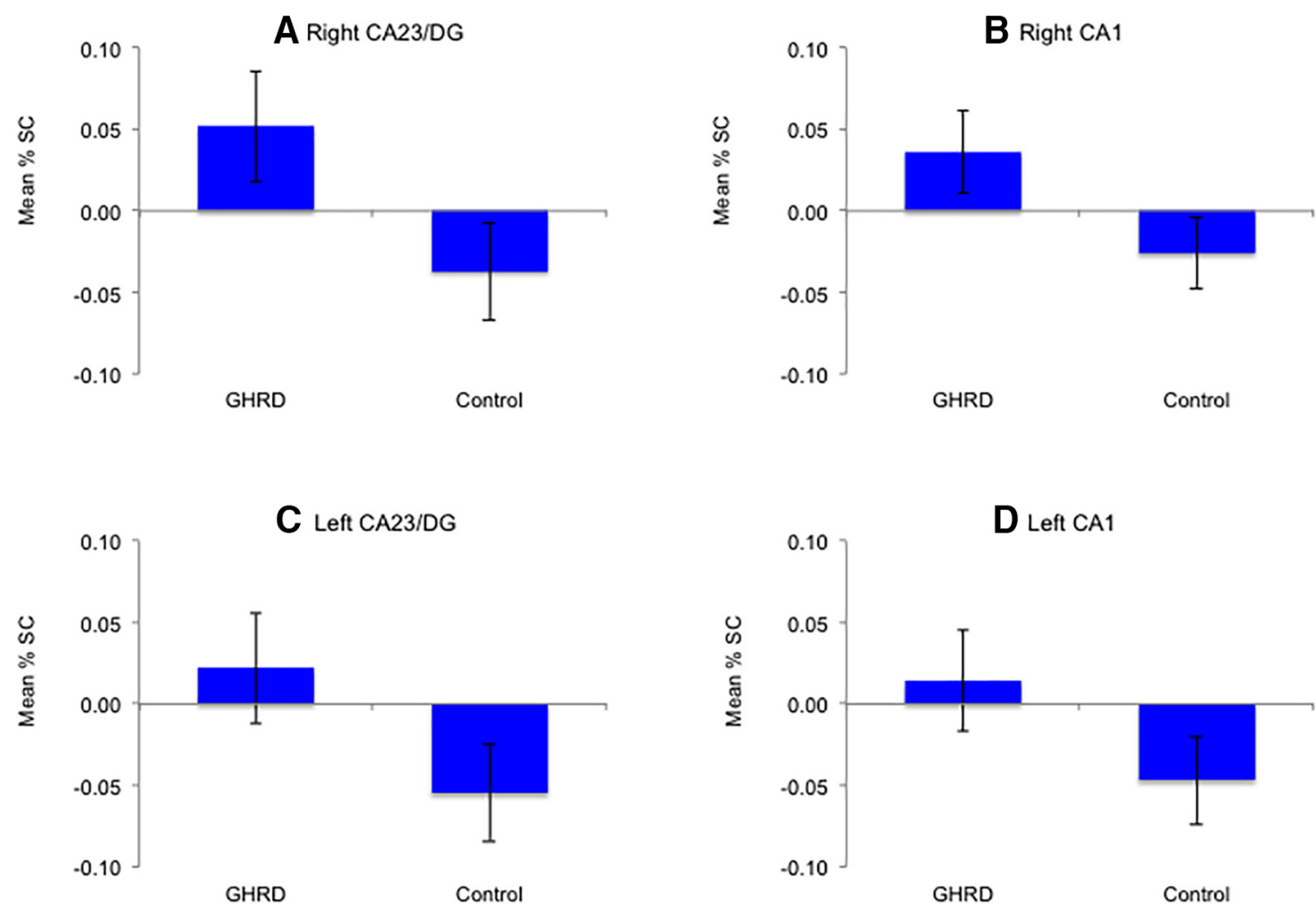

Figure 5. No significant group differences in hippocampal subfields activation. $A, B$, Relative to the control group, the GHRD group showed greater activation in the right $C A 23 / D G(A)$ and the right CA1 (B) during the similar trials; however, the results did not reach significance $(p=0.113, p=0.136$, respectively). $C, D, A$ similar pattern of group differences was observed in the left CA23/DG $(\boldsymbol{C})$ and the left CA1 (D) during the similar trials, but the results did not reach significance $(p=0.177, p=0.224$, respectively).
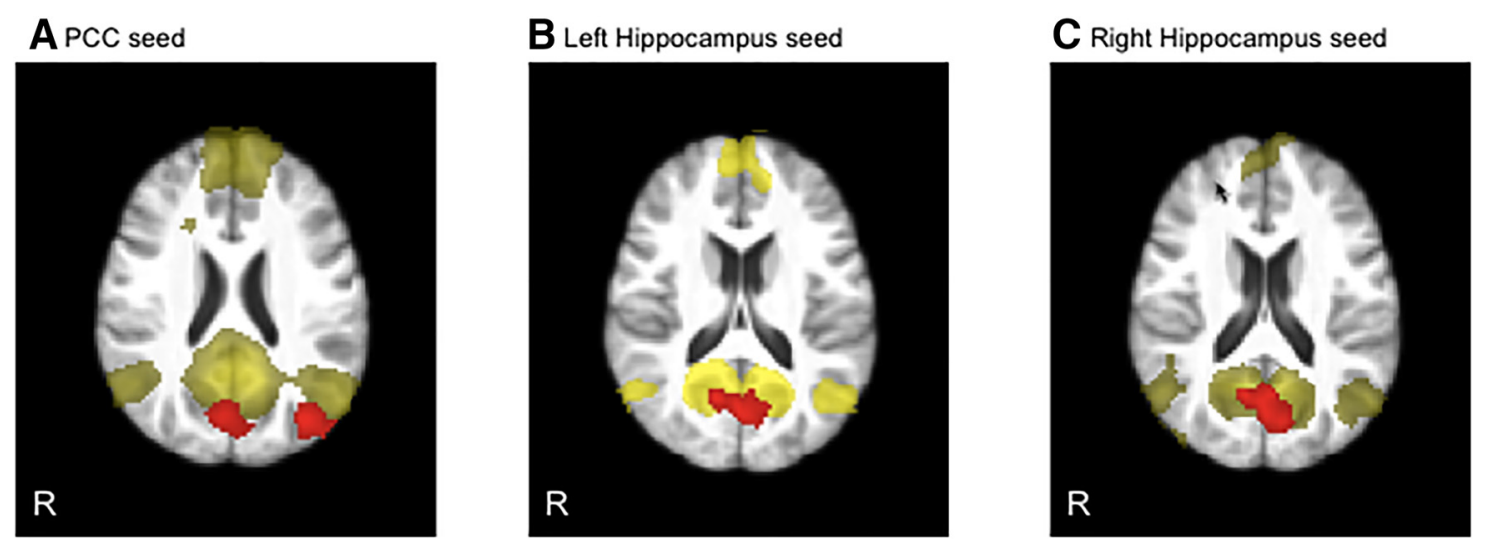

Figure 6. Brain regions showing increased functional connectivity in the GHRD group than controls at rest. The DMN is the most commonly observed resting-state network associated with internally directed mental states and cognitive function and its coordinated activity diminishes with age (Mevel et al., 2013). The yellow areas indicate the DMN defined by all participants in this study using each seed region. Relative to the control group, the GHRD group had greater functional connectivity in the precuneus (shown in red) with the PCC seed ( $A)$, the left hippocampal seed ( $B)$, and the right hippocampal seed $(\boldsymbol{C})$. With the PCC seed region, this group difference was also observed in the left occipital cortex.

mance on the pattern separation task $\left(R^{2}[\right.$ full model including age, sex, education, ICV, right hippocampus-precuneus connectivity $]=0.65 ; p[$ full model $]=0.003 ; p[$ partial contribution of precuneus-right hippocampus connectivity $]=0.021)$. Functional connectivity between the precuneus and the other two seed regions (i.e., PCC and left hippocampus) was not significantly associated with performance, but was in the same positive direction ( $p[$ partial contribution of precuneus-PCC connectivity and precuneus-right hippocampus connectivity] $=0.052$ and 0.154 , respectively). Therefore, the increased functional connectivity found in the GHRD participants may indeed suggest more efficient brain networks consistent with healthy brain function.
The E180 in the GHR in the Ecuadorian cohort results in a clinical phenotype of severe short stature and obesity, accompanied by low circulating concentrations of IGF-1 and insulin and enhanced insulin sensitivity (Rosenfeld et al., 1994; Buckway et al., 2002). Global disruption of the IGF-1 gene in mice results in disproportionate decreases in DG volume and brain myelination deficits (Beck et al., 1995). However, GHRD mutations do not appear to interfere with most of the normal production and function of autocrine and paracrine IGF-1 throughout the body because there is no evidence of defects in wound healing or immune function (Emmerson et al., 2012; Ni et al., 2013). GHRD-associated IGF-1 deficiency and related enhanced insulin 
sensitivity downregulate the expression of signaling genes, with central pro-aging roles including Tor-S6K and PKA. This may contribute to its anti-aging effect and may explain some of our results here. In fact, GHRD mice hold record longevity extension (Bartke and Brown-Borg, 2004) and GHRD mice and humans appear to be protected from cancer and diabetes (GuevaraAguirre et al., 2011, 2015), indicating that impaired function of the GHR is associated with protective effects against selected agerelated diseases. Recently, we demonstrated that diets that reduce GHR-IGF-1 signaling promoted stem-cell-based regeneration in the immune and nervous systems (Cheng et al., 2014). The use of a periodic fasting mimicking diet, which reduces insulin and IGF-1 and increases IGFBP1, promoted neural stem-cell-based regeneration as well as improved cognitive performance in aged mice (Brandhorst et al., 2015). GHRD mutations promoted a similar stem-cell-based regeneration in the hematopoietic system, suggesting that GHR-IGF1-insulin axis may influence regeneration processes significantly in multiple systems (Cheng et al., 2014).

Overall, our findings are consistent with prior studies demonstrating enhanced cognition in Ames dwarf and GHR knock-out mice (Kinney et al., 2001a, 2001b; Kinney-Forshee et al., 2004; Sharma et al., 2010). Although the mechanism for this protection is largely unknown, it could involve increased number or activity of neural stem cells and elevated regenerative function and/or increased sensitivity to IGF-1 analogously to the insulin sensitization effect that we have demonstrated recently for GHRD subjects (Guevara-Aguirre et al., 2015). Larger studies allowing for comparisons across age groups would be helpful in assessing the effect of the diminished GHR signaling on cognitive and neurological aging. Further studies are also warranted to determine whether dietary, pharmacological, or other interventions that alter brain GHR function and improve IGF- 1 and insulin sensitivity can delay cognitive aging.

\section{References}

Armstrong E, Schleicher A, Omran H, Curtis M, Zilles K (1995) The ontogeny of human gyrification. Cereb Cortex 5:56-63. CrossRef Medline

Avants BB, Yushkevich P, Pluta J, Minkoff D, Korczykowski M, Detre J, Gee JC (2010) The optimal template effect in hippocampus studies of diseased populations. Neuroimage 49:2457-2466. CrossRef Medline

Bakker A, Kirwan CB, Miller M, Stark CE (2008) Pattern separation in the human hippocampal CA3 and dentate gyrus. Science 319:1640-1642. CrossRef Medline

Barnes J, Ridgway GR, Bartlett J, Henley SM, Lehmann M, Hobbs N, Clarkson MJ, MacManus DG, Ourselin S, Fox NC (2010) Head size, age and gender adjustment in MRI studies: a necessary nuisance? Neuroimage 53: 1244-1255. CrossRef Medline

Bartke A, Brown-Borg H (2004) Life extension in the dwarf mouse. Curr Top Dev Biol 63:189-225. CrossRef Medline

Beck KD, Powell-Braxton L, Widmer HR, Valverde J, Hefti F (1995) Igf1 gene disruption results in reduced brain size, CNS hypomyelination, and loss of hippocampal granule and striatal parvalbumin-containing neurons. Neuron 14:717-730. CrossRef Medline

Brandhorst $S$ et al. (2015) A periodic diet that mimics fasting promotes multi-system regeneration, enhanced cognitive performance, and healthspan. Cell Metab 22:86-99. CrossRef Medline

Buckway CK, Selva KA, Pratt KL, Tjoeng E, Guevara-Aguirre J, Rosenfeld RG (2002) Insulin-like growth factor binding protein-3 generation as a measure of GH sensitivity. J Clin Endocrinol Metab 87:4754-4765. CrossRef Medline

Carro E, Nuñez A, Busiguina S, Torres-Aleman I (2000) Circulating insulinlike growth factor I mediates effects of exercise on the brain. J Neurosci 20:2926-2933. Medline

Carro E, Trejo JL, Gomez-Isla T, LeRoith D, Torres-Aleman I (2002) Serum insulin-like growth factor I regulates brain amyloid-beta levels. Nat Med 8:1390-1397. CrossRef Medline
Cheng CM, Cohen M, Wang J, Bondy CA (2001) Estrogen augments glucose transporter and IGF1 expression in primate cerebral cortex. FASEB J 15:907-915 CrossRef Medline

Cheng CW, Adams GB, Perin L, Wei M, Zhou X, Lam BS, Da Sacco S, Mirisola M, Quinn DI, Dorff TB, Kopchick JJ, Longo VD (2014) Prolonged fasting reduces IGF-1/PKA to promote hematopoietic-stem-cellbased regeneration and reverse immunosuppression. Cell Stem Cell 14: 810-823. CrossRef Medline

Clemmons DR (1997) Insulin-like growth factor binding proteins and their role in controlling IGF actions. Cytokine Growth Factor Rev 8:45-62. CrossRef Medline

Colcombe SJ, Erickson KI, Scalf PE, Kim JS, Prakash R, McAuley E, Elavsky S, Marquez DX, Hu L, Kramer AF (2006) Aerobic exercise training increases brain volume in aging humans. J Gerontol A Biol Sci Med Sci 61:1166-1170. CrossRef Medline

Dale AM, Fischl B, Sereno MI (1999) Cortical surface-based analysis. I. Segmentation and surface reconstruction. Neuroimage 9:179-194. CrossRef Medline

Deijen JB, Arwert LI, Drent ML (2011) The GH/IGF-I axis and cognitive changes across a 4-year period in healthy adults. ISRN Endocrinol 2011: 249421. CrossRef Medline

Emmerson E, Campbell L, Davies FC, Ross NL, Ashcroft GS, Krust A, Chambon P, Hardman MJ (2012) Insulin-like growth factor-1 promotes wound healing in estrogen-deprived mice: new insights into cutaneous IGF-1R/ERalpha cross talk. J Invest Dermatol 132:2838-2848. CrossRef Medline

Erickson KI, Voss MW, Prakash RS, Basak C, Szabo A, Chaddock L, Kim JS, Heo S, Alves H, White SM, Wojcicki TR, Mailey E, Vieira VJ, Martin SA, Pence BD, Woods JA, McAuley E, Kramer AF (2011) Exercise training increases size of hippocampus and improves memory. Proc Natl Acad Sci U S A 108:3017-3022. CrossRef Medline

Fischl B, Dale AM (2000) Measuring the thickness of the human cerebral cortex from magnetic resonance images. Proc Natl Acad Sci U S A 97: 11050-11055. CrossRef Medline

Flöel A, Ruscheweyh R, Krüger K, Willemer C, Winter B, Völker K, Lohmann H, Zitzmann M, Mooren F, Breitenstein C, Knecht S (2010) Physical activity and memory functions: are neurotrophins and cerebral gray matter volume the missing link? Neuroimage 49:2756-2763. CrossRef Medline

Frisoni GB et al.; EADC-ADNI Working Group on The Harmonized Protocol for Manual Hippocampal Volumetry and for the Alzheimer's Disease Neuroimaging Initiative (2015) The EADC-ADNI Harmonized Protocol for manual hippocampal segmentation on magnetic resonance: evidence of validity. Alzheimers Dement 11:111-125. CrossRef Medline

Greicius MD, Srivastava G, Reiss AL, Menon V (2004) Default-mode network activity distinguishes Alzheimer's disease from healthy aging: evidence from functional MRI. Proc Natl Acad Sci U S A 101:4637-4642. CrossRef Medline

Greicius MD, Flores BH, Menon V, Glover GH, Solvason HB, Kenna H, Reiss AL, Schatzberg AF (2007) Resting-state functional connectivity in major depression: abnormally increased contributions from subgenual cingulate cortex and thalamus. Biol Psychiatry 62:429-437. CrossRef Medline

Guevara-Aguirre J, Balasubramanian P, Guevara-Aguirre M, Wei M, Madia F, Cheng CW, Hwang D, Martin-Montalvo A, Saavedra J, Ingles S, de Cabo R, Cohen P, Longo VD (2011) Growth hormone receptor deficiency is associated with a major reduction in pro-aging signaling, cancer, and diabetes in humans. Sci Transl Med 3:70ra13. CrossRef Medline

Guevara-Aguirre J, Rosenbloom AL, Balasubramanian P, Teran E, GuevaraAguirre M, Guevara C, Procel P, Alfaras I, De Cabo R, Di Biase S, Narvaez L, Saavedra J, Longo VD (2015) GH receptor deficiency in ecuadorian adults is associated with obesity and enhanced insulin sensitivity. J Clin Endocrinol Metab 100:2589-2596. CrossRef Medline

Guzowski JF, Knierim JJ, Moser EI (2004) Ensemble dynamics of hippocampal regions CA3 and CA1. Neuron 44:581-584. CrossRef Medline

Hedden T, Van Dijk KR, Becker JA, Mehta A, Sperling RA, Johnson KA, Buckner RL (2009) Disruption of functional connectivity in clinically normal older adults harboring amyloid burden. J Neurosci 29:1268612694. CrossRef Medline

Im K, Lee JM, Lyttelton O, Kim SH, Evans AC, Kim SI (2008) Brain size and cortical structure in the adult human brain. Cereb Cortex 18:2181-2191. CrossRef Medline 
Jones DK, Knösche TR, Turner R (2013) White matter integrity, fiber count, and other fallacies: the do's and don'ts of diffusion MRI. Neuroimage 73:239-254. CrossRef Medline

Kinney-Forshee BA, Kinney NE, Steger RW, Bartke A (2004) Could a deficiency in growth hormone signaling be beneficial to the aging brain? Physiol Behav 80:589-594. CrossRef Medline

Kinney BA, Coschigano KT, Kopchick JJ, Steger RW, Bartke A (2001a) Evidence that age-induced decline in memory retention is delayed in growth hormone resistant GH-R-KO (Laron) mice. Physiol Behav 72:653-660. CrossRef Medline

Kinney BA, Meliska CJ, Steger RW, Bartke A (2001b) Evidence that Ames dwarf mice age differently from their normal siblings in behavioral and learning and memory parameters. Horm Behav 39:277-284. CrossRef Medline

Kranzler JH, Rosenbloom AL, Martinez V, Guevara-Aguirre J (1998) Normal intelligence with severe insulin-like growth factor I deficiency due to growth hormone receptor deficiency: a controlled study in a genetically homogeneous population. J Clin Endocrinol Metab 83:1953-1958. CrossRef Medline

Lang PJ, Bradley MM, Cuthbert BN (2005) International Affective Picture System (IAPS): affective ratings of pictures and instruction manual (Technical Report A-6). Gainesville, FL: University of Florida.

Lee PD, Giudice LC, Conover CA, Powell DR (1997) Insulin-like growth factor binding protein-1: recent findings and new directions. Proc Soc Exp Biol Med 216:319-357. CrossRef Medline

Mevel K, Landeau B, Fouquet M, La Joie R, Villain N, Mézenge F, Perrotin A, Eustache F, Desgranges B, Chételat G (2013) Age effect on the default mode network, inner thoughts, and cognitive abilities. Neurobiol Aging 34:1292-1301. CrossRef Medline

Muzumdar RH, Ma X, Fishman S, Yang X, Atzmon G, Vuguin P, Einstein FH, Hwang D, Cohen P, Barzilai N (2006) Central and opposing effects of IGF-I and IGF-binding protein-3 on systemic insulin action. Diabetes 55:2788-2796. CrossRef Medline

Ni F, Sun R, Fu B, Wang F, Guo C, Tian Z, Wei H (2013) IGF-1 promotes the development and cytotoxic activity of human NK cells. Nat Commun 4:1479. CrossRef Medline

Pajonk FG, Wobrock T, Gruber O, Scherk H, Berner D, Kaizl I, Kierer A, Müller S, Oest M, Meyer T, Backens M, Schneider-Axmann T, Thornton AE, Honer WG, Falkai P (2010) Hippocampal plasticity in response to exercise in schizophrenia. Arch Gen Psychiatry 67:133-143. CrossRef Medline

Ransome MI, Goldshmit Y, Bartlett PF, Waters MJ, Turnley AM (2004) Comparative analysis of CNS populations in knockout mice with altered growth hormone responsiveness. Eur J Neurosci 19:2069-2079. CrossRef Medline

Rosenfeld RG, Rosenbloom AL, Guevara-Aguirre J (1994) Growth hormone $(\mathrm{GH})$ insensitivity due to primary GH receptor deficiency. Endocr Rev 15:369-390. CrossRef Medline

Sagi Y, Tavor I, Hofstetter S, Tzur-Moryosef S, Blumenfeld-Katzir T, Assaf Y
(2012) Learning in the fast lane: new insights into neuroplasticity. Neuron 73:1195-1203. CrossRef Medline

Sharma S, Haselton J, Rakoczy S, Branshaw S, Brown-Borg HM (2010) Spatial memory is enhanced in long-living Ames dwarf mice and maintained following kainic acid induced neurodegeneration. Mech Ageing Dev 131: 422-435. CrossRef Medline

Shevah O, Kornreich L, Galatzer A, Laron Z (2005) The intellectual capacity of patients with Laron syndrome (LS) differs with various molecular defects of the growth hormone receptor gene: correlation with CNS abnormalities. Horm Metab Res 37:757-760. CrossRef Medline

Spielman LJ, Little JP, Klegeris A (2014) Inflammation and insulin/IGF-1 resistance as the possible link between obesity and neurodegeneration. J Neuroimmunol 273(1-2):8-21.

Takao H, Hayashi N, Inano S, Ohtomo K (2011) Effect of head size on diffusion tensor imaging. Neuroimage 57:958-967. CrossRef Medline

Thomason ME, Thompson PM (2011) Diffusion imaging, white matter, and psychopathology. Annu Rev Clin Psychol 7:63-85. CrossRef Medline

Trejo JL, Carro E, Torres-Aleman I (2001) Circulating insulin-like growth factor I mediates exercise-induced increases in the number of new neurons in the adult hippocampus. J Neurosci 21:1628-1634. Medline

Trejo JL, Llorens-Martín MV, Torres-Alemán I (2008) The effects of exercise on spatial learning and anxiety-like behavior are mediated by an IGF-I-dependent mechanism related to hippocampal neurogenesis. Mol Cell Neurosci 37:402-411. CrossRef Medline

Tustison NJ, Avants BB, Cook PA, Zheng Y, Egan A, Yushkevich PA, Gee JC (2010) N4ITK: improved N3 bias correction. IEEE Trans Med Imaging 29:1310-1320. CrossRef Medline

Westlye ET, Lundervold A, Rootwelt H, Lundervold AJ, Westlye LT (2011) Increased hippocampal default mode synchronization during rest in middle-aged and elderly APOE epsilon4 carriers: relationships with memory performance. J Neurosci 31:7775-7783. CrossRef Medline

Worsley KJ (2001) Statistical analysis of activation images. In: Functional MRI: an introduction to methods (Jezzard P, Matthews PM, Smith SM, eds), pp 251-270. New York: OUP.

Xie L, Antonow-Schlorke I, Schwab M, McDonald TJ, Nathanielsz PW, Li C (2013) The frontal cortex IGF system is down regulated in the term, intrauterine growth restricted fetal baboon. Growth Horm IGF Res 23: 187-192. CrossRef Medline

Yassa MA, Lacy JW, Stark SM, Albert MS, Gallagher M, Stark CE (2011) Pattern separation deficits associated with increased hippocampal CA3 and dentate gyrus activity in nondemented older adults. Hippocampus 21:968-979. CrossRef Medline

Zhou Y, Xu BC, Maheshwari HG, He L, Reed M, Lozykowski M, Okada S, Cataldo L, Coschigamo K, Wagner TE, Baumann G, Kopchick JJ (1997) A mammalian model for Laron syndrome produced by targeted disruption of the mouse growth hormone receptor/binding protein gene (the Laron mouse). Proc Natl Acad Sci U S A 94:13215-13220. CrossRef Medline 